\title{
$10 \%$ chlorhexidine varnish did not reduce caries in an adult population
}

\section{Abstracted from}

\section{Papas AS, Vollmer WM, Gullion CM, et al}

PACS Collaborative Group. Efficacy of chlorhexidine varnish for the prevention of adult caries: a randomized trial. J Dent Res. 2012; 91: 150-155. Epub 2011 Dec 7.

Address for correspondence William.vollmer, Kaiser Permanente Center for Health Research, 3800 North Interstate Avenue, Portland, OR 97227, USA.

Email william.vollmer@kpchr.org

\section{Question: Does chlorhexidine varnish reduce dental caries in at-risk adults over a period of 13 months?}

Design A multi-centre, placebo-controlled, double-blind, randomised clinical trial involving 983 adults (aged 18-80 years old).

Intervention The test group received chlorhexidine diacetate $10 \%$ weight per volume $(\mathrm{w} / \mathrm{v})$ dental coating and the control group received a placebo coating. Coatings were applied weekly for four weeks and a fifth time six months later.

Outcome measure The Pitts and Fyffe taxonomy ${ }^{1}$ was used to measure caries progression or reversal, which identifies three stages of lesion on coronal surfaces. The primary outcome was the total net increment in $\mathrm{D}_{1-2} \mathrm{FS}$; secondary outcomes included the cumulative net $D_{1-2} F S$ increment and the total crude $D_{1-2} F S$ increment and the cumulative crude $D_{1-2} \mathrm{FS}$ increment.

Results No significant difference was seen between the treatment and placebo groups over a 13 month study period.

Conclusions Ten percent chlorhexidine diacetate coating did not show a reduction in caries in an adult population.

\section{Commentary}

Dental caries remains one of the most prevalent chronic diseases despite it being largely preventable. Individuals are susceptible throughout their life course; therefore it is essential to conduct research into secondary prevention and the management of early, potentially reversible carious lesions in the adult population. There is a large body of evidence to support the use of fluoride varnish in the prevention of dental caries, particularly focussed on children and adolescents, ${ }^{2}$ but not so much on the use of chlorhexidine varnishes. A recent systematic review, conducted by James et al. considered the caries-preventive effect of chlorhexidine varnish in children and adolescents ${ }^{3}$ and concluded that the evidence was inconclusive.

This paper is one of a number of papers produced by the research group testing the efficacy of chlorhexidine diacetate $10 \%$ per volume coating in adults aged between 18 and 80 years old.

Whilst the particular paper did not go into the full methodology of the research, fuller details of the approach can be found in Vollmer's paper, ${ }^{4}$ demonstrating the double blinded randomised controlled trial with clear inclusion and exclusion criteria.

The use of the stratified randomisation ensured equal numbers of participants with similar characteristics were recruited into the different groups, and it was useful to see that the study population was diverse and representative, with a wide age range and residing in areas with different fluoride exposures. The authors mentioned that the weight of the vial was measured pre- and post- application of both the chlorhexidine and control coating. Further information into the analysis of the dosage would have further supported the robustness of the trial.

It is interesting to note that the authors used Pitts and Fyffe taxonomy and considered the transition from uncavitated to cavitated lesions at the $\mathrm{D}_{1}$ level. There was mention of the ICDAS (International Caries Detection and Assessment System; www.icdas. org) which is a more sensitive tool, but they did acknowledge the difficulties of clinically detecting caries at an early stage.

The results demonstrated stringent analysis, using the intention to treat analysis, with good follow up at 13 months, and were similar to the findings in previous studies using $10 \%$ chlorhexidine varnish for coronal caries. ${ }^{5}$ It could be questioned whether the 13 month follow-up period was too short a time frame but this has to be weighed up against the drop out rate and also whether a varnish containing a higher concentration of chlorhexidine be used.

In terms of influencing evidence-based practice, this study does not support the application of chlorhexidine varnish at this concentration to reduce caries in the adult population.

\section{Practice points}

- Chlorhexidine varnish is not a prevention approach that should be considered for the not at risk population

- Prevention in adults must not be ignored as the problem could potentially escalate with the ageing population retaining more natural teeth.

- An evidence-based approach is key to breaking the caries cycle.

\section{Emma O'Keefe}

Department of Public Health, NHS Fife, Leven, Scotland, UK

1. Pitts NB, Fyffe HE. The effect of varying diagnostic thresholds upon clinical caries data for a low prevalence group. J Dent Res. 1988; 67: 592-596

2. Marinho VC, Higgins JP, Logan S, Sheiham A. Fluoride varnishes for preventing dental caries in children and adolescents. Cochrane Database Syst Rev. 2002; (3): CD002279. Review

3. James P, Parnell C, Whelton $\mathrm{H}$. The caries-preventive effect of chlorhexidine varnish in children and adolescents: a systematic review. Caries Res. 2010; 44: 333-340

4. Vollmer WM, Papas AS, Bader JD, Maupome G, Gullion CM, Hollis JF, Snyder IJ, Fellows JL, Laws RL, White BA. Design of the Prevention of Adult Caries Study (PACS): A randomized clinical trial assessing the effect of a chlorhexidine dental coating for the prevention of adult caries. BMC Oral Health 2010; 10: 23

5. Banting DW, Papas A, Clark DC, Proskin HM, Schultz M, Perry R. The effectiveness of $10 \%$ chlorhexidine varnish treatment on dental caries incidence in adults with dry mouth. Gerodontology. 2000; 17: 67-76

Evidence-Based Dentistry (2012) 13, 45. doi:10.1038/sj.ebd.6400857 